\title{
PREVALENCIA DE INFECCIÓN TUBERCULOSA LATENTE EN TRABAJADORES DE SALUD DE ESTABLECIMIENTOS DEL PRIMER NIVEL DE ATENCIÓN. LIMA, PERÚ
}

\author{
Mirtha Gabriela Soto Cabezas ${ }^{1,2}$, Cesar Vladimir Munayco Escate ${ }^{1,3}$, Jaime Chávez Herrera ${ }^{4}$, \\ Sonia Llanet López Romero², David Moore ${ }^{5}$
}

\begin{abstract}
RESUMEN
Con el propósito de disponer de información sobre los riesgos para infección por tuberculosis, la Dirección General de Epidemiología del Ministerio de Salud, desarrolló una vigilancia centinela en establecimientos de salud de la Provincia constitucional del Callao, dicha vigilancia incluyó el diagnóstico de infección tuberculosa latente (ITL) mediante la aplicación del método IGRA. El objetivo del presente estudio fue estimar la prevalencia de ITL en trabajadores de salud de un área con alta carga de enfermedad de tuberculosis. La prevalencia de ITL en trabajadores de salud fue $56 \%$. En trabajadores con más de 10 años de servicio la prevalencia se incrementó a $63 \%$ y en trabajadores con más de 35 años de servicio se encontraron prevalencias entre 58 y $60 \%$. Existe una alta prevalencia de ITL en trabajadores de salud de establecimientos del primer nivel de atención, identificándose al mayor tiempo de servicio, como uno de los principales factores de riesgo.
\end{abstract}

Palabras clave: Infección tuberculosa latente, Personal de Salud, Tuberculosis (fuente: DeCS BIREME)

\section{PREVALENCE OF LATENT TUBERCULOSIS INFECTION IN HEALTH WORKERS FROM PRIMARY HEALTH CARE CENTERS IN LIMA, PERU}

\begin{abstract}
To evaluate the risk of tuberculosis (TB) infection, the General Directorate of Epidemiology of the Ministry of Health developed a sentinel surveillance system in health centers located in the Constitutional Province of Callao. This surveillance system included the diagnosis of latent tuberculosis infection (LTI) using interferon gamma release assays. The objective of the present study was to estimate the prevalence of LTI in health workers in a region with a high burden of TB. The prevalence of LTI in health workers was $56 \%$. The prevalence of $L T I$ was $63 \%$ in workers with more than 10 years of service and $58-60 \%$ in workers with more than 35 years of service. The prevalence of LTI in health workers from primary health care centers was high, and longer service time was one of the main risk factors.
\end{abstract}

Keywords: Latent Tuberculosis, Health personnel, Tuberculosis (source: MeSH NLM)

\section{INTRODUCCIÓN}

La tuberculosis (TB) constituye un riesgo laboral importante en los trabajadores de salud (TS) de zonas urbanas con alta carga de enfermedad tuberculosa y donde, muchas veces, las medidas de prevención y control son limitadas ${ }^{(1,2)}$.

En Perú, la tuberculosis es una enfermedad endémica, según el último informe de la Organización Panamericana de la Salud es el segundo país con la mayor carga de TB en la región de América Latina y el Caribe ${ }^{(3)}$. Para el año 2015 se reportó una incidencia de 88 casos nuevos de TB por cada 100 mil habitantes, siendo Lima y Callao las ciudades que concentran casi el $60 \%$ de todos los casos del país ${ }^{(4)}$. El promedio de TS afectados por TB en los últimos 10 años en nuestro país, varió entre 200 antes del 2010 y casi 300 para el año 2015 y, de estos, casi el 60\% se reportaron en las provincias de Lima y Callao ${ }^{(5)}$.

El estado de infección tuberculosa latente (ITL) en una persona constituye un riesgo importante en el desarrollo de la enfermedad tuberculosa, a nivel mundial se ha estimado una prevalencia de ITL en TS de $54 \%$ en países de bajos y medianos ingresos, con rango de 33 a $79 \%$; por otro lado,

\footnotetext{
Centro Nacional de Epidemiología, Prevención y Control de Enfermedades. Lima, Perú

Facultad de Medicina. Universidad Peruana Cayetano Heredia. Lima, Perú

Facultad de Salud Pública y Administración. Universidad Peruana Cayetano Heredia. Lima, Perú

Dirección Regional de Salud del Callao. Callao, Perú.

5 Facultad de Ciencias y Filosofía, Universidad Peruana Cayetano Heredia. Lima, Perú

Recibido: 02/08/2017 Aprobado: 30/11/2017 En línea: 04/12/2017
}

Citar como: Soto Cabezas MG, Munayco Escate CV, Chávez Herrera J, López Romero SL, Moore D. Prevalencia de infección tuberculosa latente en trabajadores de salud de establecimientos del primer nivel de atención. Lima, Perú. Rev Peru Med Exp Salud Publica. 2017;34(4):649-54. doi: 10.17843/ rpmesp.2017.344.3035 
se describió que el riesgo de adquirir un ITL en los TS se ve influenciado por la prevalencia local de la TB en comunidad, el tipo de establecimiento de salud (ES) y la cantidad de pacientes que se atienden, las áreas o servicios donde laboran y el grupo ocupacional, así como la eficacia de las medidas de control de infecciones de TB ${ }^{(1,2,6,7)}$.

En el Perú se han realizado algunos estudios que han evidenciado altas prevalencias de ITL en TS, pero estos se han realizado, principalmente, en hospitales o en estudiantes de carreras de ciencias de la salud ${ }^{(8-12)}$. Sin embargo, no se han desarrollado estudios en ES del primer nivel de atención y son estos, precisamente, donde se administran gratuitamente los tratamientos a los pacientes con TB.

Por otro lado, la técnica habitual para el diagnóstico de ITLes la prueba de la tuberculina (PT), sin embargo, el mayor problema de esta prueba es su poca especificidad. En la actualidad existen otros métodos diagnósticos basados en la cuantificación in vitro de la respuesta inmune celular, los llamados ensayos de liberación de interferón gamma (IGRA, por su traducción del inglés interferon gamma release assays) ${ }^{(13,14)}$.

El objetivo del presente estudio fue estimar la prevalencia de ITL en TS de establecimientos del primer nivel de atención, utilizando los datos de la vigilancia centinela realizada en los ES del primer nivel de atención de la RED Bonilla de la Dirección Regional de Salud (DIRESA) del Callao, así como describir los factores de riesgo que mejor predicen la ITL en esta población.

\section{MATERIALES Y MÉTODOS}

Se realizó un estudio descriptivo utilizando una fuente de datos secundario, recolectados en la vigilancia centinela de TB en TS de los ES de la Red Bonilla de la DIRESA Callao.

En este contexto, la Dirección General de Epidemiología (DGE) del Ministerio de Salud, en coordinación con la DIRESA del Callao, desarrollaron entre marzo y junio del año 2008 una vigilancia centinela de la ITL en TS de todos los ES de la Red de Salud Bonilla, dicha vigilancia incluyó una medición basal con el diagnóstico de ITL mediante la aplicación del método IGRA, utilizando la técnica Quantiferon-in-the-tube Gold testing (QFT-G-IT), la cual fue procesada en los laboratorios de la Universidad Peruana Cayetano Heredia.

Además, a cada trabajador se le aplicó una ficha epidemiológica, con el propósito de disponer de información sobre los factores de riesgo para un manejo individual de los casos. En esta vigilancia se incluyó como TS, a todo personal (profesional, técnico u otro) que laboraba en los ES, bajo cualquier tipo de contrato; se incluyeron también

\section{MENSAJES CLAVE}

Motivación para realizar el estudio. La tuberculosis es un problema de salud pública, que afecta no solo a la población general, sino también a los encargados de combatirla.

Principales hallazgos. Se encontró que la prevalencia de infección tuberculosis latente (ITL) en personal de salud fue de $56 \%$ y que esta incrementa directamente con el tiempo de servicio.

Implicancias. La prevalencia de ITL es alta en todo el personal de salud, inclusive en aquellos que no realizan labor asistencial o que no están en contacto directo con el paciente, lo que implica que las medidas de control de infecciones deben aplicarse por todo el personal de salud, asistencial o no, principalmente en zonas como Callao, que tiene una alta carga de tuberculosis en la comunidad.

a estudiantes de carreras de ciencias de la salud, que realizaban rotaciones o prácticas en los establecimientos de salud.

Se revisó la base de datos de la vigilancia centinela, incluyéndose en el estudio las siguientes variables: resultado de la prueba IGRA, edad, sexo, lugar de nacimiento, años de servicio en el establecimiento de salud, ocupación, antecedentes de enfermedad tuberculosa, contacto con pacientes afectados por TB. En el estudio solo se incluyeron los casos que tuvieron resultados de la prueba IGRA positivo o negativo. Todos los pacientes con resultado IGRA positivo tuvieron baciloscopías negativas y radiografías de tórax normales.

Se realizó un análisis descriptivo basado en la obtención de frecuencias absolutas y relativas, medidas de tendencia central y de dispersión. Se determinó la prevalencia de ITL de acuerdo al resultado positivo obtenido con IGRA en el grupo poblacional estudiado y se estratificó de acuerdo a las variables del estudio. Para identificar los factores de riesgo que mejor predicen la ITL se realizó un análisis bivariado y multivariado utilizando regresión logística, el orden de la inclusión de las variables en el modelo final se hizo de acuerdo al valor $p$ obtenido en el análisis bivariado tomando como punto de corte el valor $p<0,2$. El análisis estadístico fue realizado con el programa estadístico STATA versión 11,0.

Con relación a los aspectos éticos, se obtuvo la base de datos omitiendo los nombres y datos personales de los participantes, con fines de garantizar la confidencialidad de la información, además, se contó con la autorización institucional para la difusión de los datos.

\section{RESULTADOS}

Se incluyeron en el estudio a 150 trabajadores de salud. La edad promedio de los TS fue de 43 años (mínimo 19 
y máximo 68 años) y el $77 \%$ fueron de sexo femenino, un alto porcentaje $(71,3 \%)$ nacieron en las provincias de Lima y Callao. Los técnicos en enfermería (35,3\%), personal administrativo (20\%), enfermeros y obstetras $(14,7 \%)$ y médicos $(6,7 \%)$ fueron los grupos ocupacionales más frecuentes, $55 \%$ de los TS tenían un tiempo de servicio mayor a 10 años. El $81,3 \%$ de los TS refirieron haber atendido (en fase de diagnóstico o tratamiento) a pacientes con tuberculosis, el $36,7 \%$ de los TS refirieron haber tenido contacto con algún familiar o amigo cercano con diagnóstico de TB; 3,3\% (5 TS) manifestaron haber tenido TB anteriormente (Tabla 1).

La prevalencia de ITL (prueba IGRA QFT-G-IT positiva) en la población estudiada fue $56 \%$ (intervalo de confianza (IC) 95\%: 46,7\% - 63,4\%). La prevalencia de ITL fue mayor en

Tabla 1. Características generales de trabajadores de salud de establecimientos del primer nivel de atención. Callao, Perú (año 2008)

\begin{tabular}{|c|c|}
\hline Variables & $n=150$ \\
\hline Edad $\pm \mathrm{DE}$ (mínimo-máximo) (años) & $43 \pm 11(19-68)$ \\
\hline \multicolumn{2}{|l|}{ Sexo $(\%)$} \\
\hline Femenino & $116(77,3)$ \\
\hline Masculino & $34(22,7)$ \\
\hline \multicolumn{2}{|l|}{ Lugar de nacimiento (\%) } \\
\hline Lima y Callao & $107(71,3)$ \\
\hline Otros departamentos & $43(28,7)$ \\
\hline \multicolumn{2}{|l|}{ Lugar de residencia (\%) } \\
\hline Distritos de Callao & $107(71,3)$ \\
\hline Distritos de Lima & $43(28,7)$ \\
\hline Antecedente de enfermedad tuberculosa (\%) & $5(3,3)$ \\
\hline \multicolumn{2}{|l|}{ Ocupación/Profesión (\%) } \\
\hline Médico & $10(6,7)$ \\
\hline Enfermera/Obstetra & $22(14,7)$ \\
\hline Técnicos en enfermería & $53(35,2)$ \\
\hline Otros profesionales de salud* & $16(10,7)$ \\
\hline Personal administrativo & $30(20,0)$ \\
\hline Otros & $19(12,7)$ \\
\hline \multicolumn{2}{|l|}{ Carga de TB en el ES donde laboran (\%) } \\
\hline $\begin{array}{l}\text { Establecimientos que reportan menos a } \\
50 \text { casos de TB por año }\end{array}$ & $98(65,3)$ \\
\hline $\begin{array}{l}\text { Establecimientos que reportan más de } 50 \\
\text { casos de TB por año }\end{array}$ & $52(34,7)$ \\
\hline $\begin{array}{l}\text { ¿En toda su carrera ha atendido a pacientes } \\
\text { con TB? }\end{array}$ & $122(81,3)$ \\
\hline $\begin{array}{l}\text { Antecedente de contacto con familiares y } \\
\text { amigos con TB }\end{array}$ & $55(36,7)$ \\
\hline $\begin{array}{l}\text { Antecedente de haber tenido TB alguna vez } \\
\text { en su vida }\end{array}$ & $5(3,3)$ \\
\hline \multicolumn{2}{|l|}{ Tiempo de servicio $(n=133)$ (\%) (años) } \\
\hline$\leq 5$ & $38(28,6)$ \\
\hline $6-10$ & $22(16,5$ \\
\hline$>10$ & $73(54,9)$ \\
\hline
\end{tabular}

el sexo masculino, en los grupos de 35 años a más y en los nacidos en Lima y Callao. La prevalencia de ITL en TS se incrementó a mayor tiempo de servicio. El porcentaje de ITL según ocupación u profesión fue similar (Tabla 2).

En el análisis bivariado se observó una razón de probabilidades (OR) de 4,5 y 4,4 para los grupos de 35 a 44 y 45 a 54 años, respectivamente, comparados con el grupo más joven (19 a 24 años), esta diferencia fue significativa $(p<0,05)$. También se observó un incremento del OR a mayor tiempo de servicio. En los TS con más de 10 años de servicio el riesgo fue casi el doble que el grupo de menos de 5 años de servicio $(p=0,12)$ (Tabla 2$)$.

Por otro lado, las variables edad y tiempo de servicio estuvieron correlacionadas, por lo que se escogió para el modelo final la variable tiempo de servicio, porque está relacionada con la exposición a la tuberculosis en los servicios de salud. En el modelo final se observó un OR de 2,3 en los TS con más de 10 años de servicio comparado con los de menos de 5 años de servicio $(p=0,05)$, ajustado por sexo, lugar de nacimiento y contacto de pacientes con TB en su centro laboral (Tabla 3 ).

\section{DISCUSIÓN}

La prevalencia estimada en el presente estudio fue similar a lo reportado en otros estudios realizados en nuestro país ${ }^{(8,9)}$; $\sin$ embargo, nuestros resultados fueron obtenidos utilizando la técnica QFT-G-IT, que cuantifica la respuesta inmune celular y el resultado no es modificado por la vacunación previa con BCG, ni con infecciones por micobacterias atípicas, por lo tanto, su especificidad es mayor que la PT; además, varios estudios sobre esta técnica han demostrado que la sensibilidad de QFT-G-IT es mayor que la PT en contactos recientes de pacientes bacilíferos ${ }^{(13,15)}$.

En el estudio se exploró y describió los factores de riesgo relacionados a una mayor prevalencia de ITL en TS, observando una asociación entre un mayor tiempo de servicio y el incremento del riesgo de adquirir ITL; además, se observó el incremento de riesgo en los grupos de mayor edad, estos dos factores han sido descritos con mayor frecuencia en otros estudios ${ }^{(1,2,6)}$. Por otro lado, en Perú no se dispone de información sobre prevalencias de ITL por grupos etarios en la población general, tal vez este dato pudiera ser útil para diferenciar el riesgo en TS y en la población general.

Respecto a los grupos ocupacionales, no se evidenció diferencias significativas, sin embargo, en varios estudios se describe una asociación con los procedimientos o las áreas donde laboran, como trabajar en salas de hospitalización o de emergencia y la participación en los procedimientos como recojo de esputo y autopsias, entre otros ${ }^{(1,2,6-9)}$, estos factores no pudieron ser evaluados en este estudio. 
Tabla 2. Prevalencia y factores asociados a infección tuberculosa latente en trabajadores de salud de establecimientos del primer nivel de atención. Callao, Perú (año 2018).

\begin{tabular}{|c|c|c|c|c|c|c|}
\hline Variables & $\mathrm{n} / \mathrm{N}$ & $\%$ & IC $95 \%$ & $\begin{array}{l}\text { OR } \\
\text { crudo }\end{array}$ & IC $95 \%$ & $\begin{array}{l}\text { Valor } \\
\text { de } p\end{array}$ \\
\hline Casos de ITL (prueba IGRA positivo) & $84 / 150$ & 56,0 & $46,7-63,4$ & NA & NA & NA \\
\hline \multicolumn{7}{|l|}{ Sexo } \\
\hline Femenino & $63 / 116$ & 54,3 & $44,8-63,7$ & 1,0 & - & NA \\
\hline Masculino & $21 / 34$ & 61,7 & $39,1-75,5$ & 1,4 & $0,6-3,0$ & 0,44 \\
\hline \multicolumn{7}{|l|}{ Edad (años) } \\
\hline$<25$ & $3 / 12$ & 25,0 & $5,4-57,1$ & 1,0 & - & NA \\
\hline $25-34$ & $14 / 25$ & 56,0 & $30,6-73,2$ & 3,8 & $0,8-17,6$ & 0,09 \\
\hline $35-44$ & $24 / 40$ & 60,0 & $43,4-76,0$ & 4,5 & $1,1-19,2$ & 0,04 \\
\hline $45-54$ & $29 / 49$ & 59,2 & $43,2-72,4$ & 4,4 & $1,0-18,1$ & 0,04 \\
\hline$>55$ & $14 / 24$ & 58,3 & $36,6-77,9$ & 4,2 & $0,9-19,6$ & 0,07 \\
\hline \multicolumn{7}{|l|}{ Lugar de nacimiento } \\
\hline Lima y Callao & $64 / 107$ & 59,8 & $48,6-68,5$ & 1,7 & $0,8-3,5$ & 0,14 \\
\hline Otros departamentos & $20 / 43$ & 46,5 & $31,2-62,3$ & 1,0 & - & NA \\
\hline \multicolumn{7}{|l|}{ Lugar de residencia } \\
\hline Distritos de alto riesgo & $60 / 108$ & 55,5 & $44,3-64,2$ & 0,9 & $0,4-1,8$ & 0,71 \\
\hline Distrito de mediano o bajo riesgo & $24 / 42$ & 57,1 & $40,9-73,0$ & 1,0 & - & NA \\
\hline \multicolumn{7}{|l|}{ Ocupación/profesión } \\
\hline Médico & $6 / 10$ & 60,0 & $21,2-86,3$ & 1,7 & $0,3-7,9$ & 0,52 \\
\hline Enfermera/Obstetra & $12 / 22$ & 54,5 & $32,2-75,6$ & 1,3 & $0,4-4,6$ & 0,65 \\
\hline Otros profesionales de salud* & $9 / 16$ & 56,3 & $26,5-78,7$ & 1,4 & $0,4-5,4$ & 0,60 \\
\hline Técnicos en enfermería & $30 / 53$ & 56,6 & $42,2-70,6$ & 1,4 & $0,5-4,1$ & 0,49 \\
\hline Personal administrativo & $18 / 30$ & 60,0 & $40,6-77,3$ & 1,7 & $0,4-5,3$ & 0,39 \\
\hline Otros & $9 / 19$ & 47,4 & $21,5-69,2$ & 1,0 & - & NA \\
\hline \multicolumn{7}{|l|}{ Carga de TB en el ES donde laboran } \\
\hline $\begin{array}{l}\text { Establecimientos que reportan menos de } \\
50 \text { casos de TB por año }\end{array}$ & $54 / 98$ & 55,1 & $43,6-64,7$ & 1,0 & - & NA \\
\hline $\begin{array}{l}\text { Establecimientos que reportan más de } 50 \\
\text { casos de TB por año }\end{array}$ & $30 / 52$ & 57,7 & $42,2-70,7$ & 1,1 & $0,6-2,2$ & 0,76 \\
\hline \multicolumn{7}{|l|}{ Tiempo de servicio ( $n=128)$ (años) } \\
\hline$\leq 5$ & $18 / 38$ & 47,4 & $27,2-62,1$ & 1,0 & - & NA \\
\hline $6-10$ & $12 / 22$ & 54,5 & $32,2-75,6$ & 1,3 & $0,5-3,8$ & 0,59 \\
\hline$>10$ & $46 / 73$ & 63,0 & $50,3-73,6$ & 1,9 & $0,9-4,2$ & 0,12 \\
\hline \multicolumn{7}{|l|}{$\begin{array}{l}\text { ¿En toda su carrera ha tenido contacto } \\
\text { con pacientes con tuberculosis en el } \\
\text { Establecimiento de Salud? }\end{array}$} \\
\hline $\mathrm{Si}$ & $71 / 122$ & 58,2 & $47,8-66,4$ & 1,6 & $0,7-3,7$ & 0,26 \\
\hline No & $13 / 28$ & 46,4 & $27,5-66,1$ & 1,0 & - & NA \\
\hline \multicolumn{7}{|l|}{$\begin{array}{l}\text { Antecedente de contacto con familiares y } \\
\text { amigos con TB }\end{array}$} \\
\hline Sí & $30 / 55$ & 54,5 & $37,6-66,0$ & 0,9 & $0,5-1,8$ & 0,3 \\
\hline No & $54 / 95$ & 56,8 & $46,3-67,2$ & 1,0 & - & NA \\
\hline
\end{tabular}

NA: no aplica ref.: referencia

* Odontólogo, psicólogo, nutricionista, asistenta social, tecnólogo médico 
Tabla 3. Factores de riesgo asociados a infección tuberculosa latente según factores de riesgo evaluado, en trabajadores de salud de establecimientos del primer nivel de atención. Callao, Perú (año 2018)

\begin{tabular}{|c|c|c|c|}
\hline Variables & $\begin{array}{c}\text { OR } \\
\text { ajustado* }\end{array}$ & $\begin{array}{c}\text { IC } \\
95 \%\end{array}$ & $\begin{array}{l}\text { Valor } \\
\text { de } p\end{array}$ \\
\hline \multicolumn{4}{|l|}{ Sexo } \\
\hline Masculino & 2,2 & $0,9-5,7$ & 0,22 \\
\hline Femenino & 1,0 & - & NA \\
\hline \multicolumn{4}{|l|}{ Lugar de nacimiento } \\
\hline Lima y Callao & 1,9 & $0,9-4,2$ & 0,12 \\
\hline Otros departamentos & 1,0 & - & NA \\
\hline \multicolumn{4}{|l|}{ Tiempo de Servicio (años) } \\
\hline$\leq 5$ & 1,0 & - & NA \\
\hline $6-10$ & 1,5 & $0,5-4,4$ & 0,50 \\
\hline$>10$ & 2,3 & $1,0-5,4$ & 0,05 \\
\hline \multicolumn{4}{|c|}{$\begin{array}{l}\text { ¿En toda su carrera ha tenido contacto } \\
\text { con pacientes con tuberculosis en el } \\
\text { establecimiento de salud? }\end{array}$} \\
\hline Sí & 1,1 & $0,4-3,0$ & 0,85 \\
\hline No & 1,0 & - & NA \\
\hline
\end{tabular}

* Ajustado por sexo, lugar de nacimiento, tiempo de servicio y contacto de pacientes con TB en su centro laboral

Otro factor importante, descrito en varios estudios, es la carga de TB en la población cercana al establecimiento de salud, el estudio de Baussano et al., realizado en el año 2011, estimó que, en países con alta carga de TB, la incidencia de ITL en TS es mayor que en países de mediana y baja carga. En el presente estudio no se evidenciaron diferencias significativas entre TS de ES que atienden una alta carga de casos de TB (más de 50 casos de TB por año), probablemente porque varios de estos TS viven en distritos de alto riesgo (con incidencia de TB superiores a 50 casos por año), la probabilidad de ponerse en contacto con un paciente bacilífero con TB activa diagnosticada o no identificada es alta, no solo en el establecimiento de salud sino también en la comunidad $(1,2,6)$

La principal debilidad de este estudio fue el uso de datos de fuente secundaria, ya que no permitió explorar factores de riesgo importantes no incluidos en la base de datos, además del poco tamaño de muestra y datos incompletos en algunas variables. Sin embargo, los resultados del presente estudio son uno de los primeros reportes de este problema en trabajadores de establecimientos de salud del primer nivel de atención. Estudios previos en Perú fueron realizados en hospitales, por lo tanto, los resultados que aporta el presente estudio pueden ser de utilidad para plantear investigaciones especificas en el tema.

Finalmente, concluimos que existe una alta prevalencia de ITL en trabajadores de salud de establecimientos del primer nivel de atención de la Red de Salud Bonilla en Callao, identificándose como factor de riesgo a un mayor tiempo de servicio.

Contribuciones de autoría: MGSC, CVME, JCHE, SLLR y DM han participado en la concepción y diseño del artículo y aprobación de la versión final. Además, MGSC y CVME realizaron recolección de datos, el análisis de datos y redacción del artículo. CVME JCHE y DM en la revisión crítica del estudio.

Fuentes de financiamiento: autofinanciado.

Conflictos de interés: los Autores MGSC y CVME trabajan en la Dirección General de Epidemiología, hoy Centro Nacional de Epidemiologia, Prevención y control de Enfermedades.

\section{REFERENCIAS BIBLIOGRÁFICAS}

1. Joshi R, Reingold AL, Menzies D, Pai M. Tuberculosis among health-care workers in low- and middle-income countries: a systematic review. PLoS Med. 2006;3(12):e494.

2. Baussano I, Nunn P, Williams B, Pivetta E, Bugiani M, Scano F. Tuberculosis among health care workers. Emerg Infect Dis. $2011 \mathrm{Mar} ; 17(3): 488-94$.

3. Organización Panamericana de la Salud. La tuberculosis en la Región de las Américas: Informe Regional 2012. Epidemiología, control y financiamiento [Internet]. Washington, DC: OPS; 2013. (citado 02 enero 2014). Disponible en http://www.paho.org/hq/index.php?option $=$ com_docman\&task=doc_view\&gi $\mathrm{d}=22954+\&$ Itemid=999999\&lang=es
4. Alarcon Guizado A. Situación actual de la gestión en el control de TB en el Perú: análisis epidemiológico y operacional [Internet]. Lima: Ministerio de Salud; 2015 [citado 12 julio 2015] Disponible en: http://190.223.45.115/newtb/Archivos/ RecursoInformacion/20150930144300. pdf

5. Soto-Cabezas MG, Chávez-Pachas AM, Arrasco-Alegre JC, Yagui-Moscoso MJA. Tuberculosis en trabajadores de salud en el Perú, 2013-2015. Rev Peru Med Exp Salud Pública. 2016;33(4):607-15.

6. Menzies D, Joshi R, Pai M. Risk of tuberculosis infection and disease associated with work in health care settings. Int J Tuberc Lung Dis. 2007;11(6):593 605 .
7. Hernández M, Casar C, García P, Morales $\mathrm{V}$, Mamani N, Gómez-Cofré $\mathrm{N}$, et al. Pesquisa de infección tuberculosa latente en personal de la salud en cuatro instituciones de salud en Santiago de Chile. Rev Chil Infectol. 2014;31(3):254-60.

8. Escombe AR, Huaroto L, Ticona E, Burgos M, Sanchez I, Carrasco L, et al. Tuberculosis transmission risk and infection control in a hospital emergency department in Lima, Peru. Int J Tuberc Lung Dis. 2010;14(9):1120-6.

9. Alonso-Echanove J, Granich RM, Laszlo A, Chu G, Borja N, Blas R, et al. Occupational transmission of Mycobacterium tuberculosis to health care workers in a university hospital in Lima, Peru. Clin Infect Dis Off Publ Infect Dis Soc Am. 2001;33(5):589-96. 
10. Bonifacio N, Saito M, Gilman RH, Leung F, Cordova Chavez N, Chacaltana Huarcaya J, et al. High risk for tuberculosis in hospital physicians, Peru. Emerg Infect Dis. 2002;8(7):747-8.

11. Hohmuth BA, Yamanija JC, Dayal AS, Nardell E, Salazar JJ, Smith Fawzi MC. Latent tuberculosis infection: risks to health care students at a hospital in Lima, Peru. Int J Tuberc Lung Dis. 2006;10(10):1146-51.

12. Pérez-Lu JE, Cárcamo CP, García PJ, Bussalleu A, Bernabé-Ortiz A. Tuberculin skin test conversion among health sciences students: A retrospective cohort study. Tuberc Edinb Scotl. 2013;93(2):257-62.

13. Arias Guillén M. Avances en el diagnóstico de la infección tuberculosa. Arch Bronconeumol. 2011;47(10):521-30.

14. Horvat RT. Review of Interferon Gamma Assays used in the Diagnosis of Tuberculosis. Clin Vaccine Immunol. 2015;22(8):845-9.

15. Gonzślez-Moreno J, García-Gasalla M, Gállego-Lezaun C, Fernández-Baca V, Mir Viladrich I, Cifuentes-Luna C, et al. Role of QuantiFERON( $\left.{ }^{\circ}\right)-\mathrm{TB}$ Gold In-
Tube in tuberculosis contact investigation: experience in a tuberculosis unit. Infect Dis (Lond). 2015;47(4):244-51.

Correspondencia: Mirtha Gabriela Soto Cabezas Dirección: Calle Daniel Olaechea 199 Jesús María. Lima, Perú

Teléfono: (+51) 987133962

Correo electrónico:gamis11@gmail.com

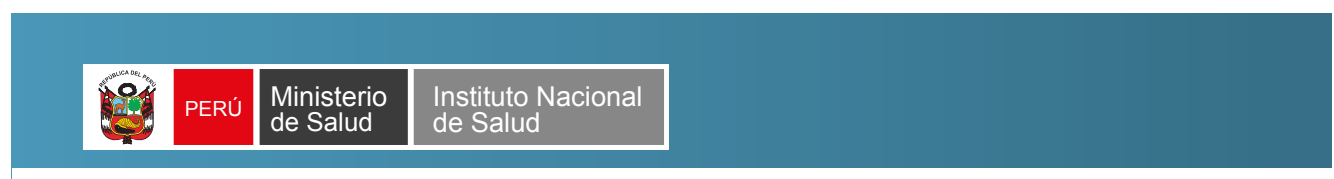

\section{Inclusión social en salud: acercando el diagnóstico de dengue a las poblaciones afectadas}

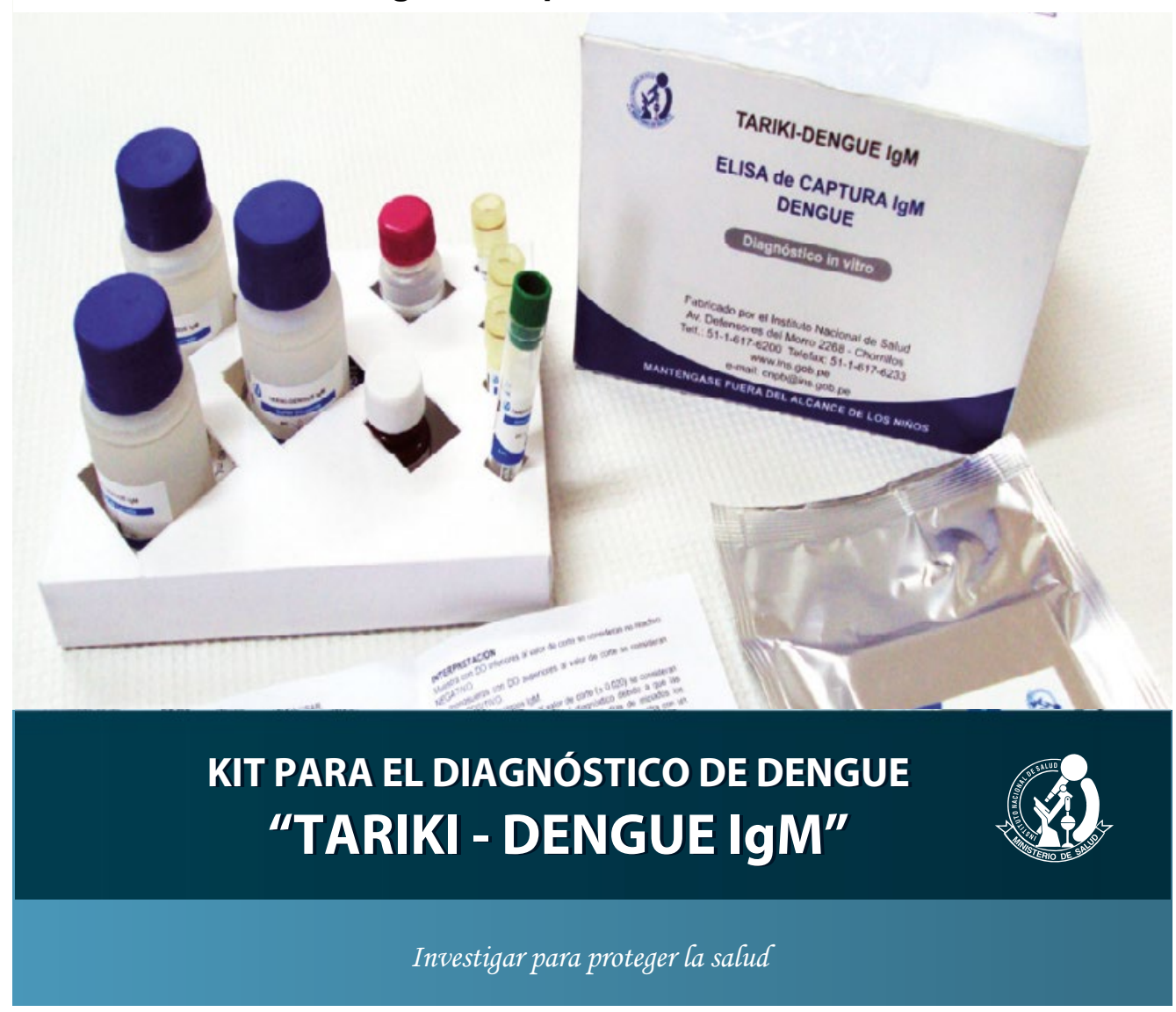

